\title{
International consensus guidance for management of myasthenia gravis
}

\section{Executive summary}

\section{OPEN}

Donald B. Sanders, MD* Gil I. Wolfe, MD* Michael Benatar, MD, $\mathrm{PhD}$

Amelia Evoli, MD

Nils E. Gilhus, MD

Isabel Illa, MD

Nancy Kuntz, MD

Janice M. Massey, MD

Arthur Melms, MD

Hiroyuki Murai, MD

Michael Nicolle, MD

Jacqueline Palace, BM, DM

David P. Richman, MD Jan Verschuuren, MD

Pushpa Narayanaswami, MBBS, DM*

Correspondence to

Dr. Sanders:

donald.sanders@duke.edu

Editorial, page 350

Supplemental data at Neurology.org

\section{ABSTRACT}

Objective: To develop formal consensus-based guidance for the management of myasthenia gravis (MG).

Methods: In October 2013, the Myasthenia Gravis Foundation of America appointed a Task Force to develop treatment guidance for MG, and a panel of 15 international experts was convened. The RAND/UCLA appropriateness methodology was used to develop consensus guidance statements. Definitions were developed for goals of treatment, minimal manifestations, remission, ocular MG, impending crisis, crisis, and refractory MG. An in-person panel meeting then determined 7 treatment topics to be addressed. Initial guidance statements were developed from literature summaries. Three rounds of anonymous e-mail votes were used to attain consensus on guidance statements modified on the basis of panel input.

Results: Guidance statements were developed for symptomatic and immunosuppressive treatments, IV immunoglobulin and plasma exchange, management of impending and manifest myasthenic crisis, thymectomy, juvenile MG, MG associated with antibodies to muscle-specific tyrosine kinase, and $M G$ in pregnancy.

Conclusion: This is an international formal consensus of $M G$ experts intended to be a guide for clinicians caring for patients with MG worldwide. Neurology ${ }^{\circledR}$ 2016;87:419-425

\section{GLOSSARY}

AChR $=$ acetylcholine receptor; $\mathbf{C h E I}=$ cholinesterase inhibitor; $\mathbf{C T C A E}=$ Common Terminology Criteria for Adverse Events; FDA = Food and Drug Administration; IS = immunosuppressive; IVIg = IV immunoglobulin; JMG = juvenile myasthenia gravis; MG = myasthenia gravis; MGFA = Myasthenia Gravis Foundation of America; MMS = minimal manifestation status; MuSK = muscle-specific tyrosine kinase; PIS = Post-Intervention Status; PLEX = plasma exchange; RAM = RAND/ UCLA Appropriateness Method; $\mathbf{R C T}=$ randomized controlled trial.

Acquired myasthenia gravis $(\mathrm{MG})$ is a disorder of neuromuscular transmission, resulting from binding of autoantibodies to components of the neuromuscular junction, most commonly the acetylcholine receptor (AChR). The incidence ranges from 0.3 to 2.8 per $100,000,{ }^{1}$ and it is estimated to affect more than 700,000 people worldwide.

The increasing use of immunomodulating therapies has been a major factor in improving the prognosis for patients with MG in recent years. ${ }^{2}$ The various treatment options must be weighed in the context of individual patient factors.

Why do we need MG guidance treatment statements? Although there is widespread agreement on the use of many treatments for MG, there is no internationally accepted standard of care. Because MG is heterogeneous, no one

*These authors contributed equally to this work.

From the Department of Neurology (D.B.S., J.M.M.), Duke University Medical Center, Durham, NC; Department of Neurology (G.I.W.), University at Buffalo School of Medicine and Biomedical Sciences, State University of New York; Department of Neurology (M.B.), University of Miami, Miller School of Medicine, FL; Department of Neurology (A.E.), Catholic University, Rome, Italy; Department of Clinical Medicine (N.E.G.), University of Bergen, Norway; Department of Neurology (I.I.), Hospital Santa Creu i Sant Pau, Universitat Autònoma de Barcelona; CIBERER U762 (I.I.), Barcelona, Spain; Departments of Pediatrics and Neurology (N.K.), Northwestern Feinberg School of Medicine, Chicago, IL; Neurologische Klinik (A.M.), Universitätsklinikum Erlangen; Hertie Institute for Clinical Research (A.M.), University of Tübingen, Germany; Department of Neurological Therapeutics (H.M.), Graduate School of Medical Sciences, Kyushu University, Fukuoka, Japan; Department of Clinical Neurological Sciences (M.N.), Western University, London, Canada; Department of Clinical Neurology (J.P.), John Radcliffe Hospital, Oxford University Hospitals Trust, Oxford, UK; Department of Neurology (D.P.R.), University of California, Davis, CA; Department of Neurology (J.V.), Leiden University Medical Centre, Leiden, the Netherlands; and Department of Neurology, Beth Israel Deaconess Medical Center/Harvard Medical School (P.N.), Boston, MA. Go to Neurology.org for full disclosures. Funding information and disclosures deemed relevant by the authors, if any, are provided at the end of the article. The Article Processing Charge was paid by the Myasthenia Gravis Foundation of America.

This is an open access article distributed under the terms of the Creative Commons Attribution-NonCommercial-NoDerivatives License 4.0 (CC BY-NC-ND), which permits downloading and sharing the work provided it is properly cited. The work cannot be changed in any way or used commercially. 
treatment approach is best for all patients. Few physicians treat enough patients with MG to be comfortable with all available treatments. Given its heterogeneity, the few randomized controlled trials (RCTs) in MG have limited generalizability, while uncontrolled trials are limited by potential bias. Hence, an effort to develop consensus among international experts was undertaken to guide clinicians worldwide on the multifaceted approach to managing MG. This summary condenses the extensive background information in the full guidance statements, available on the Neurology ${ }^{\circledR}$ Web site at Neurology.org.

Panel constitution and method of expert consensus. In October 2013, a Task Force of the Myasthenia Gravis Foundation of America (MGFA) convened a panel of 15 international experts in MG to develop treatment guidance statements based on formalized consensus. The panel was chosen to represent the breadth of knowledge and experience and a wide variety of opinions from MG experts internationally.

Development of preliminary definitions. The panel initially voted on definitions that formed the foundation for subsequent guidance treatment statements: goals of treatment, remission, ocular MG, impending and manifest myasthenic crises, and refractory MG.

The Task Force co-chairs (D.B.S., G.I.W.) drafted initial definitions based on available literature. ${ }^{3}$ These were sent by e-mail to the panelists, who were asked to vote yes or no on each, and to provide modifications if they did not agree. Panelists were instructed not to discuss the definitions among themselves, and to send their votes only to the facilitator (P.N.). A simple consensus was used ( $\geq 80 \%$ of panelists voting yes).

Definitions not achieving consensus were modified based on the panelists' suggestions and the modified definitions and discussions were shared with the panel for subsequent voting rounds.

Development of guidance treatment statements. The following were agreed upon a priori:

1. Treatment costs and availability would not be considered, as it is not possible to make international consensus statements specific for all countries.

2. Clinical examination is assumed to have been performed by physicians skilled in the evaluation of neuromuscular disease.

3. The MGFA Clinical Classification, including remission, refers to the state of the patient at the time of evaluation.

A formal systematic review of the literature was not performed. The Task Force co-chairs and facilitator drafted initial guidance statements based on literature cited in recent national and regional $\mathrm{MG}$ treatment guidelines, ${ }^{4-9}$ supplemented by other literature.

Guidance statements were developed for the following:

1. Symptomatic and immunosuppressive treatments

2. IV immunoglobulin (IVIg) and plasma exchange (PLEX)

3. Impending and manifest myasthenic crisis

4. Thymectomy

5. Juvenile MG (JMG)

6. MG with antibodies to muscle-specific tyrosine kinase (MuSK-MG)

7. $M G$ in pregnancy

Voting process for consensus guidance treatment statements. We used the RAND/UCLA Appropriateness Method (RAM) for formal consensus to quantify agreement. ${ }^{10}$ RAM uses a multi-round modified Delphi process to obtain a quantitative assessment that reflects the judgment of an expert group. Appropriateness refers to the relative benefit vs harm of the intervention. We obtained anonymous votes and feedback on each draft statement from the panelists, who rated each for appropriateness on a 9point scale (1-3 inappropriate, 4-6 uncertain, and 79 appropriate). Panelists responded by e-mail to the facilitator, who tallied the votes and collated the discussions. Following each round of voting, statements were modified by the Task Force cochairs and facilitator based on the panel feedback. Statements that did not achieve consensus within 3 rounds were excluded.

For statements on symptomatic and IS therapies and thymectomy, an initial round of e-mail voting was followed by a meeting in Durham, North Carolina, on March 1, 2014. During this meeting, statements that had undergone prior voting by e-mail were refined with panel input, and a second round of voting was completed. All subsequent voting was by e-mail.

The level of appropriateness and presence of agreement were determined for each statement as per RAM. ${ }^{10}$

RESULTS All definitions below obtained simple consensus and all guidance statements below were agreed upon as being appropriate by the panel. Literature summaries and tables for medication dosing guidance and medication cautions are available as supplemental data at Neurology.org.

\section{Preliminary definitions.}

1. Goals for the treatment of MG. MGFA Task Force Post-Intervention Status (PIS) classification Minimal Manifestation Status (MMS) or better, ${ }^{3}$ with no more than grade 1 Common Terminology Criteria for Adverse Events (CTCAE) medication side effects. ${ }^{11}$ 
MMS: The patient has no symptoms or functional limitations from MG but has some weakness on examination of some muscles. This class recognizes that some patients who otherwise meet the definition of remission have mild weakness.

CTCAE grade 1 medication side effects: asymptomatic or only mild symptoms; intervention not indicated.

2. Definition of remission. The patient has no symptoms or signs of MG. Weakness of eyelid closure is accepted, but there is no weakness of any other muscle on careful examination. Patients taking cholinesterase inhibitors (ChEIs) every day with reasonable evidence to support symptomatic benefit are therefore excluded from this category.

3. Definition of ocular MG (based on dysfunction due to $M G$ at a specified point in time, and not dependent upon the duration of disease). MGFA Class $\mathrm{I}^{3}$ : Any ocular muscle weakness. May have weakness of eye closure. Strength in all other facial, bulbar, and limb muscles is normal. (It is recognized that some patients report fatigue when strength testing is normal. The physician should use clinical judgment in attributing fatigue to generalized MG in the absence of objective nonocular weakness).

4. Definition of impending myasthenic crisis. Rapid clinical worsening of MG that, in the opinion of the treating physician, could lead to crisis in the short term (days to weeks).

5. Definition of manifest myasthenic crisis (the concept of crisis focuses on the clinical implications-it represents a serious, life-threatening, rapid worsening of MG and potential airway compromise from ventilatory or bulbar dysfunction). MGFA Class $\mathrm{V}^{3}$ : Worsening of myasthenic weakness requiring intubation or noninvasive ventilation to avoid intubation, except when these measures are employed during routine postoperative management (the use of a feeding tube without intubation places the patient in MGFA Class IVB ${ }^{3}$ ).

6. Definition of refractory $M G$. PIS $^{3}$ is unchanged or worse after corticosteroids and at least 2 other IS agents, used in adequate doses for an adequate duration, with persistent symptoms or side effects that limit functioning, as defined by patient and physician.

Consensus guidance treatment statements.

Symptomatic and IS treatment of MG.

1. Pyridostigmine should be part of the initial treatment in most patients with MG. Pyridostigmine dose should be adjusted as needed based on symptoms. The ability to discontinue pyridostigmine can be an indicator that the patient has met treatment goals and may guide the tapering of other therapies. Corticosteroids or IS therapy should be used in all patients with MG who have not met treatment goals after an adequate trial of pyridostigmine.
2. A nonsteroidal IS agent should be used alone when corticosteroids are contraindicated or refused. A nonsteroidal IS agent should be used initially in conjunction with corticosteroids when the risk of steroid side effects is high based on medical comorbidities. A nonsteroidal IS agent should be added to corticosteroids when:

a. Steroid side effects, deemed significant by the patient or the treating physician, develop;

b. Response to an adequate trial (table e-1) of corticosteroids is inadequate; or

c. The corticosteroid dose cannot be reduced due to symptom relapse.

3. Nonsteroidal IS agents that can be used in MG include azathioprine, cyclosporine, mycophenolate mofetil, methotrexate, and tacrolimus. The following factors should be considered in selecting among these agents:

a. There is widespread variation in practice with respect to choice of IS agent since there is little literature comparing them.

b. Expert consensus and some RCT evidence support the use of azathioprine as a first-line IS agent in MG.

c. Evidence from RCTs supports the use of cyclosporine in MG, but potential serious adverse effects and drug interactions limit its use.

d. Although available RCT evidence does not support the use of mycophenolate and tacrolimus in MG, both are widely used, and one or both are recommended in several national MG treatment guidelines. $^{4-7}$

4. Patients with refractory MG should be referred to a physician or a center with expertise in management of MG. In addition to the previously mentioned IS agents, the following therapies may also be used in refractory MG:

a. Chronic IVIg and chronic PLEX (see IVIg and PLEX, no. 6);

b. Cyclophosphamide;

c. Rituximab, for which evidence of efficacy is building, but for which formal consensus could not be reached.

5. IS agent dosage and duration of treatment

a. Once patients achieve treatment goals, the corticosteroid dose should be gradually tapered. In many patients, continuing a low dose of corticosteroids long-term can help to maintain the treatment goal.

b. For nonsteroidal IS agents, once treatment goals have been achieved and maintained for 6 months to 2 years, the IS dose should be tapered slowly to the minimal effective amount. Dosage adjustments should be made no more frequently than every 3-6 months (table e-1). 
c. Tapering of IS drugs is associated with risk of relapse, which may necessitate upward adjustments in dose. The risk of relapse is higher in patients who are symptomatic, or after rapid taper.

d. It is usually necessary to maintain some immunosuppression for many years, sometimes for life.

6. Patients must be monitored for potential adverse effects and complications from IS drugs. Changing to an alternative IS agent should be considered if adverse effects and complications are medically significant or create undue hardship for the patient.

\section{IVIg and PLEX.}

1. PLEX and IVIg are appropriately used as shortterm treatments in patients with MG with lifethreatening signs such as respiratory insufficiency or dysphagia; in preparation for surgery in patients with significant bulbar dysfunction; when a rapid response to treatment is needed; when other treatments are insufficiently effective; and prior to beginning corticosteroids if deemed necessary to prevent or minimize exacerbations.

2. The choice between PLEX and IVIg depends on individual patient factors (e.g., PLEX cannot be used in patients with sepsis and IVIg cannot be used in renal failure) and on the availability of each.

3. IVIg and PLEX are probably equally effective in the treatment of severe generalized MG.

4. The efficacy of IVIg is less certain in milder MG or in ocular MG.

5. PLEX may be more effective than IVIg in MuSKMG.

6. The use of IVIg as maintenance therapy can be considered for patients with refractory MG or for those in whom IS agents are relatively contraindicated.

Impending and manifest myasthenic crisis. Impending and manifest myasthenic crisis are emergent situations requiring aggressive management and supportive care.

Although cholinergic crises are now rare, excessive ChEI cannot be completely excluded as a cause of clinical worsening. Also, ChEIs increase airway secretions, which may exacerbate breathing difficulties.

PLEX and IVIg are the mainstay of management in myasthenic crisis.

1. Impending crisis requires hospital admission and close observation of respiratory and bulbar function, with the ability to transfer to an intensive care unit if it progresses to manifest crisis. Myasthenic crisis requires admission to an intensive care or step-down unit to monitor for or manage respiratory failure and bulbar dysfunction.

2. PLEX and IVIg are used as short-term treatment for impending and manifest myasthenic crisis and in patients with significant respiratory or bulbar dysfunction. Corticosteroids or other IS agents are often started at the same time to achieve a sustained clinical response. (Because corticosteroids may cause transient worsening of myasthenic weakness, it may be appropriate to wait several days for PLEX or IVIg to have a beneficial effect before starting corticosteroids).

3. Although clinical trials suggest that IVIg and PLEX are equally effective in the treatment of impending or manifest myasthenic crisis, expert consensus suggests that PLEX is more effective and works more quickly. The choice between the 2 therapies depends on patient comorbidity (e.g., PLEX cannot be used in sepsis and IVIg is contraindicated in hypercoagulable states, renal failure, or hypersensitivity to immunoglobulin) and other factors, including availability. A greater risk of hemodynamic and venous access complications with PLEX should also be considered in the decision (many complications of PLEX are related to route of access and may be minimized by using peripheral rather than central venous access).

\section{Thymectomy in $M G$.}

1. In non-thymomatous MG, thymectomy is performed as an option to potentially avoid or minimize the dose or duration of immunotherapy, or if patients fail to respond to an initial trial of immunotherapy or have intolerable side-effects from that therapy. Because of the long delay in onset of effect, thymectomy for MG is an elective procedure. It should be performed when the patient is stable and deemed safe to undergo a procedure where postoperative pain and mechanical factors can limit respiratory function.

2. The value of thymectomy in the treatment of prepubertal patients with MG is unclear, but thymectomy should be considered in children with generalized AChR antibody-positive MG:

a. If the response to pyridostigmine and IS therapy is unsatisfactory; or

b. In order to avoid potential complications of IS therapy.

For children diagnosed with seronegative generalized MG, the possibility of a congenital myasthenic syndrome or other neuromuscular condition should be entertained, and evaluation at a center specializing in neuromuscular diseases is of value prior to thymectomy.

3. With rare exceptions, all patients with MG with thymoma should undergo surgery to remove the tumor. Removal of the thymoma is performed to rid the patient of the tumor and may not produce improvement in MG. All thymus tissue should be 
removed along with the tumor. Further treatment of thymoma will be dictated by histologic classification and degree of surgical excision. Incompletely resected thymomas should be managed after surgery with an interdisciplinary treatment approach (radiotherapy, chemotherapy).

4. In elderly or multimorbid patients with thymoma, palliative radiation therapy can be considered in the appropriate clinical setting. Small thymomas may be followed without treatment unless they are enlarging or become symptomatic.

5. Endoscopic and robotic approaches to thymectomy are increasingly performed and have a good track record for safety in experienced centers. Data from randomized, controlled comparison studies are not available. Based on comparisons across studies, less invasive thymectomy approaches appear to yield similar results to more aggressive approaches.

6. Thymectomy may be considered in patients with generalized MG without detectable AChR antibodies if they fail to respond adequately to IS therapy, or to avoid/minimize intolerable adverse effects from IS therapy. Current evidence does not support an indication for thymectomy in patients with MuSK, LRP4, or agrin antibodies.

Juvenile MG (see also Thymectomy in MG, no. 2).

1. Children with acquired autoimmune ocular MG are more likely than adults to go into spontaneous remission. Thus, young children with only ocular symptoms of MG can be treated initially with pyridostigmine. Immunotherapy can be initiated if goals of therapy are not met.

2. Children are at particular risk of steroid side effects, including growth failure, poor bone mineralization, and susceptibility to infection, due in part to a delay in live vaccinations. Long-term treatment with corticosteroids should use the lowest effective dose to minimize side effects.

3. Maintenance PLEX or IVIg are alternatives to IS drugs in JMG.

\section{MG with MuSK antibodies.}

1. Many patients with MuSK-MG respond poorly to ChEIs, and conventional pyridostigmine doses frequently induce side effects.

2. Patients with MuSK-MG appear to respond well to corticosteroids and to many steroid-sparing IS agents. They tend to remain dependent on prednisone despite concomitant treatment with steroidsparing agents.

3. MuSK-MG responds well to PLEX, while IVIg seems to be less effective.

4. Rituximab should be considered as an early therapeutic option in patients with MuSK-MG who have an unsatisfactory response to initial immunotherapy.

\section{MG in pregnancy.}

1. Planning for pregnancy should be instituted well in advance to allow time for optimization of myasthenic clinical status and to minimize risks to the fetus.

2. Multidisciplinary communication among relevant specialists should occur throughout pregnancy, during delivery, and in the postpartum period.

3. Provided that their myasthenia is under good control before pregnancy, the majority of women can be reassured that they will remain stable throughout pregnancy. If worsening occurs, it may be more likely during the first few months after delivery.

4. Oral pyridostigmine is the first-line treatment during pregnancy. IV ChEIs may produce uterine contractions and should not be used during pregnancy.

5. Thymectomy should be postponed until after pregnancy as benefit is unlikely to occur during pregnancy.

6. Chest CT without contrast can be performed safely during pregnancy, although the risks of radiation to the fetus need to be carefully considered. Unless there is a compelling indication, postponement of diagnostic CT until after delivery is preferable.

7. Prednisone is the IS agent of choice during pregnancy.

8. Current information indicates that azathioprine and cyclosporine are relatively safe in expectant mothers who are not satisfactorily controlled with or cannot tolerate corticosteroids. Current evidence indicates that mycophenolate mofetil and methotrexate increase the risk of teratogenicity and are contraindicated during pregnancy. (These agents previously carried Food and Drug Administration [FDA] Category C (cyclosporine), D (azathioprine and mycophenolate mofetil), and X (methotrexate) ratings. The FDA has recently discontinued this rating system, and replaced it with a summary of the risks of using a drug during pregnancy and breastfeeding, along with supporting data and "relevant information to help health care providers make prescribing and counseling decisions"12). Although this statement achieved consensus, there was a strong minority opinion against the use of azathioprine in pregnancy. Azathioprine is the nonsteroidal IS of choice for MG in pregnancy in Europe but is considered high risk in the United States. This difference is based on a small number of animal studies and case reports.

9. PLEX or IVIg are useful when a prompt, although temporary, response is required during pregnancy. Careful consideration of both maternal and fetal issues, weighing the risks of these treatments against the requirement for use during pregnancy and their potential benefits, is required. 
10. Spontaneous vaginal delivery should be the objective and is actively encouraged.

11. Magnesium sulfate is not recommended for management of eclampsia in MG because of its neuromuscular blocking effects; barbiturates or phenytoin usually provide adequate treatment.

12. All babies born to myasthenic mothers should be examined for evidence of transient myasthenic weakness, even if the mother's myasthenia is well-controlled, and should have rapid access to neonatal critical care support.

DISCUSSION We have developed international guidance statements for the management of JMG and adult MG. We utilized recent national guidelines and a regional European guideline to assemble a foundation of literature, supplementing their comprehensive literature reviews with additional articles identified by panelists. After reaching agreement on the treatment goals, a 3-round anonymous modified Delphi voting process was used to obtain consensus on guidance statements.

A limitation of consensus-based processes is that subconscious or conscious selection of like-minded panel members may result in opinions that are not representative of MG experts. This issue was addressed by selecting an international panel with variations in practice and by using a formal consensus process.

Recognizing the variability of practice patterns and availability of treatment modalities, these statements are not absolute recommendations for management, but are intended as a guide for the clinician. They are also not intended for establishing payment policies or drug tiering by payers.

This is a living document that will require updates as the MG treatment theater continues to evolve. Despite the limitations of consensus-based methods, these guidance statements reflect an up-to-date expert consensus to guide clinicians worldwide who strive to optimize function and quality of life for their patients with MG, especially for those who practice in parts of the world that do not have the resources to develop local treatment guidelines. Any future trial of treatment that provides relevant information will merit review of these guidance statements.

\section{AUTHOR CONTRIBUTIONS}

D.B. Sanders: study concept and design, grant application and management, acquisition of data, analysis or interpretation of data, drafting/ revising the manuscript, critical revision of the manuscript for important intellectual content, study supervision. G.I. Wolfe: study concept and design, acquisition of data, analysis or interpretation of data, drafting/ revising the manuscript, critical revision of the manuscript for important intellectual content, study supervision. M. Benatar: acquisition of data, drafting/revising the manuscript, critical revision of the manuscript for important intellectual content. A. Evoli: acquisition of data, drafting/ revising the manuscript, critical revision of the manuscript for important intellectual content. N.E. Gilhus: acquisition of data, critical revision of the manuscript for important intellectual content. I. Illa: acquisition of data, critical revision of the manuscript for important intellectual content. N. Kuntz: acquisition of data, drafting/revising the manuscript, critical revision of the manuscript for important intellectual content. J.M. Massey: acquisition of data, drafting/revising the manuscript, critical revision of the manuscript for important intellectual content. A. Melms: acquisition of data, critical revision of the manuscript for important intellectual content. H. Murai: acquisition of data, critical revision of the manuscript for important intellectual content. J. Palace: acquisition of data, drafting/revising the manuscript, critical revision of the manuscript for important intellectual content. M. Nicolle: acquisition of data, drafting/revising the manuscript, critical revision of the manuscript for important intellectual content. D.P. Richman: acquisition of data, critical revision of the manuscript for important intellectual content. J. Verschuuren: acquisition of data, critical revision of the manuscript for important intellectual content. P. Narayanaswami: study concept and design, acquisition of data, analysis or interpretation of data, drafting/revising the manuscript, critical revision of the manuscript for important intellectual content, study supervision.

\section{ACKNOWLEDGMENT}

The authors thank MGFA Board member Jurgen Venitz, MD, PhD, for providing insight from the patients' perspective.

\section{STUDY FUNDING}

Supported by a grant from the Myasthenia Gravis Foundation of America (MGFA).

\section{DISCLOSURE}

D. Sanders: consultant for Accordant Health Services, Cytokinetics, GlaxoSmithKline, and Jacobus Pharmaceutical Co. G. Wolfe: advisory board for Grifols, Baxter, CSL Behring, and Syntimmune; and research support from Alexion. M. Benatar: research support from the FDA (R01FD003710), NIH (U01NS084495), and Alexion. A. Evoli: scientific award jury member for Grifols. N. Gilhus: speaker's honoraria from Octopharma, Baxter, and Merck Serono. I. Illa: research funding from Grifols and speaking fees and travel grants from Genzyme and Pfizer; and consultant for Alexion, UCB, and Grifols. N. Kuntz and J. Massey report no disclosures relevant to the manuscript. A. Melms: advisory board, UCB. H. Murai: research funding from Biogen, Novartis, Bayer, Tanabe Mitsubishi, and the Japan Blood Products Organization. J. Palace: consultant for Merck Serono, Biogen Idec, Novartis, Teva, Chugai Pharma, Alexion, and Bayer Schering; research funding from Merck Serono, Novartis, Biogen Idec, and Bayer Schering. M. Nicolle reports no disclosures relevant to the manuscript. D. Richman: research funding from the Muscular Dystrophy Association, Myasthenia Gravis Foundation of California, and the Myasthenia Gravis Foundation of Illinois. J. Verschuuren: research support by NIH, FP7 European grant (\#602420), consultant for Tyr pharma, and arGEN-X. The LUMC receives royalties from IBL for antibody tests. P. Narayanaswami: serves on the Medical and Scientific Advisory Board, Myasthenia Gravis Foundation of America (MGFA), and Medical Advisory Board, MGFA, New England Chapter. Go to Neurology.org for full disclosures.

Received August 25, 2015. Accepted in final form February 19, 2016.

\section{REFERENCES}

1. Deenen JCW, Horlings CGC, Verschuuren JJGM, Verbeek ALM. The epidemiology of neuromuscular disorders: a comprehensive overview of the literature. J Neuromuscul Dis 2015;2:73-85.

2. Grob D, Brunner NG, Namba T, Pagala M. Lifetime course of myasthenia gravis. Muscle Nerve 2008;37:141-149.

3. Jaretzki A III, Barohn RB, Ernstoff RM, et al. Myasthenia gravis: recommendations for clinical research standards: Task Force of the Medical Scientific Advisory Board of the Myasthenia Gravis Foundation of America. Neurology 2000;55:16-23. 
4. Wiendl H. Diagnostik und Therapie der Myasthenia gravis und des Lambert-Eaton-Syndroms [online]. Available at: http://www.dgn.org/leitlinien/11-leitlinien-der-dgn/3005-ll68-ll-diagnostik-und-therapie-der-myasthenia-gravis-unddes-lambert-eaton-syndroms. Accessed August 14, 2015.

5. Fuhr P, Gold R, Hohlfeld R, et al. Diagnostik und therapie der myasthenia gravis und des Lambert-Eaton Syndroms. In: Diener HC, Weimar C, Deuschl G, et al, eds. Leitlinien für Diagnostik und Therapie in der Neurologie, 5th ed. Stuttgart: Thieme; 2012:830-856.

6. Murai $H$. Japanese clinical guidelines for myasthenia gravis: putting into practice. Clin Exp Neuroimmunol 2015;6:21-31.

7. Sussman J, Farrugia ME, Maddison P, Hill M, Leite MI, Hilton-Jones D. Myasthenia gravis: association of British Neurologists' management guidelines. Pract Neurol 2015; 15:199-206.

8. Norwood F, Dhanjal M, Hill M, et al. Myasthenia in pregnancy: best practice guidelines from a UK multispecialty working group. J Neurol Neurosurg Psychiatry 2014;85: 538-543.

9. Skeie GO, Apostolski S, Evoli A, et al. Guidelines for treatment of autoimmune neuromuscular transmission disorders. Eur J Neurol 2010;17:893-902.

10. Fitch K, Bernstein SJ, Aguilar MD, et al. The RAND/UCLA Appropriateness Method User's Manual. Santa Monica, CA: RAND Corporation; 2001.

11. US Department of Health and Human Services. Common Terminology Criteria for Adverse Events (CTCAE) v4.03 [online]. Available at: http://evs.nci.nih.gov/ftp1/CTCAE/ CTCAE_4.03_2010-06-14_QuickReference_5x7.pdf. Accessed May 25, 2016.

12. US Food and Drug Administration. Pregnancy and Lactation Final Rule [online]. Available at: http://www.fda.gov/Drugs/ DevelopmentApprovalProcess/DevelopmentResources/ Labeling/ucm093307.htm. Accessed November 11, 2015.

\section{This Week's Neurology ${ }^{\circledR}$ Podcast}

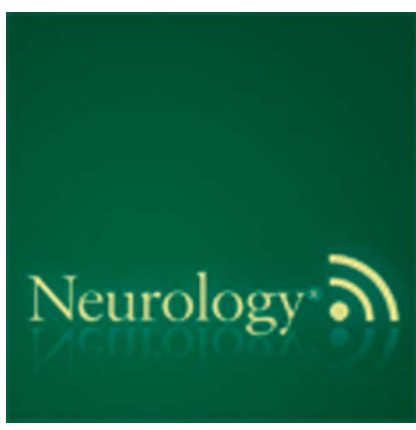

International consensus guidance for management of myasthenia gravis: Executive summary (see p. 419)

This podcast begins and closes with Dr. Robert Gross, Editor-inChief, briefly discussing highlighted articles from the July 26, 2016, issue of Neurology. In the second segment, Dr. Ted Burns talks with Dr. Donald Sanders about the international consensus guidance for the management of myasthenia gravis. Dr. Ilena George reads the e-Pearl of the week about progressive multifocal leukoencephalopathy. Dr. Tesha Monteith focuses his interview with Dr. Richard Lipton on his Neurology Year in Review plenary session at the AAN Annual Meeting discussing headache.

Disclosures can be found at Neurology.org.

At Neurology.org, click on "RSS" in the Neurology Podcast box to listen to the most recent podcast and subscribe to the RSS feed.

CME Opportunity: Listen to this week's Neurology Podcast and earn 0.5 AMA PRA Category

1 CME Credits ${ }^{\mathrm{TM}}$ by answering the multiple-choice questions in the online Podcast quiz.

\section{Get Connected. Stay Connected.}

Connect with the American Academy of Neurology's popular social media channels to stay up-todate on the latest news and breakthroughs in neurology, and network with peers and neurology thought leaders. Visit AAN.com/Connect. 


\section{Neurology}

\section{International consensus guidance for management of myasthenia gravis: Executive summary}

Donald B. Sanders, Gil I. Wolfe, Michael Benatar, et al. Neurology 2016;87;419-425 Published Online before print June 29, 2016

DOI 10.1212/WNL.0000000000002790

This information is current as of June 29, 2016

\section{Updated Information \& Services \\ Supplementary Material}

\section{References}

Citations

Subspecialty Collections

Permissions \& Licensing

Reprints including high resolution figures, can be found at: http://n.neurology.org/content/87/4/419.full

Supplementary material can be found at: http://n.neurology.org/content/suppl/2016/06/29/WNL.0000000000002 790.DC1

http://n.neurology.org/content/suppl/2016/06/29/WNL.0000000000002 790.DC2

http://n.neurology.org/content/suppl/2016/07/25/WNL.0000000000002 790.DC3

This article cites 7 articles, 3 of which you can access for free at: http://n.neurology.org/content/87/4/419.full\#ref-list-1

This article has been cited by 21 HighWire-hosted articles: http://n.neurology.org/content/87/4/419.full\#\#otherarticles

This article, along with others on similar topics, appears in the following collection(s):

\section{Myasthenia}

http://n.neurology.org/cgi/collection/myasthenia

Information about reproducing this article in parts (figures,tables) or in its entirety can be found online at:

http://www.neurology.org/about/about_the_journal\#permissions

Information about ordering reprints can be found online:

http://n.neurology.org/subscribers/advertise

Neurology ${ }^{\circledR}$ is the official journal of the American Academy of Neurology. Published continuously since 1951, it is now a weekly with 48 issues per year. Copyright (O 2016 American Academy of Neurology. All rights reserved. Print ISSN: 0028-3878. Online ISSN: 1526-632X.

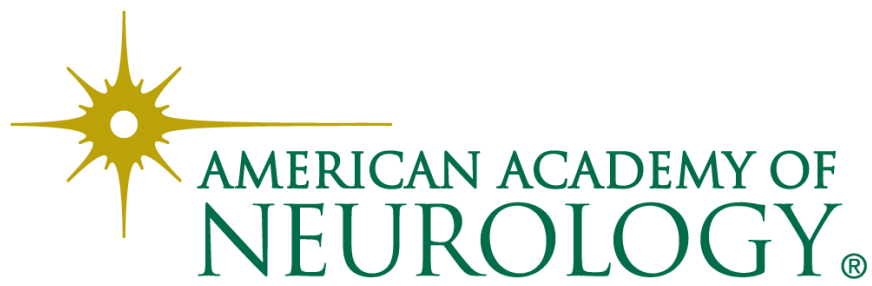

